\title{
The Effect of Plastic Deformation of Austenite on the Kinetics of Subsequent Ferrite Formation
}

\author{
David N. HANLON, ${ }^{1)}$ Jilt SIETSMA ${ }^{2)}$ and Sybrand van der ZWAAG $^{1,2)}$ \\ 1) The Netherlands Institute for Metals Research, Rotterdamseweg 137, 2628 AL Delft, the Netherlands. \\ E-mail: D.N.HANLON@TNW.TUDELFT.NL 2 2) Laboratory of Materials Science, Delft University of Technology, \\ Rotterdamseweg 137, 2628 AL Delft, the Netherlands.
}

(Received on January 29, 2001; accepted in final form on May 15, 2001)

\begin{abstract}
A modeling approach to account for the effect of deformation on the austenite to ferrite transformation is described. In this approach the stored energy of deformation resulting from the formation of a dislocation substructure is considered to result in an elevation of the driving force for transformation. A scheme which accounts for the competition between softening and transformation processes is also described. Model data illustrating the effects of discrete dislocations and cell structures, with and without concurrent recovery are presented. Comparison of modeled and experimental transformation curves indicates that deformation affects the transformation principally via a reduction in the undercooling required for nucleation rather than via an acceleration of the growth kinetics.
\end{abstract}

KEY WORDS: steels; austenite; phase transformations; recovery; hot deformation.

\section{Introduction}

Modelling approaches which have been used to describe the austenite to ferrite transformation in steels include both empirical and physically based methodologies. The physically based models proposed to date fall into three basic categories. Diffusion control models assume that the growth of ferrite is governed by the rate of carbon diffusion in the austenitic phase. ${ }^{1,2)}$ Interface mobility control models assume that the mobility of the interface itself determines the rate of transformation. ${ }^{3)}$ Mixed mode models attempt to account for both the effects of diffusion and interface mobility. ${ }^{4)}$ Common to all of these approaches is the assumption that transformation occurs from a starting microstructure of recrystallised or undeformed austenite. In the case of the traditional hot rolling process for plain carbon steels this is a reasonable assumption. However, in the case of modern steel grades and rolling schedules, situations may arise which invalidate this assumption. For instance, the drive to hot roll to thinner gauges may lead, deliberately or otherwise, to finishing temperatures below the transformation start temperature $\left(\mathrm{Ar}_{3}\right)$, and rolling therefore takes place in the intercritical regime. Furthermore, for modern HSLA steel grades the kinetics of recrystallisation may be so severely retarded that a fully recrystallised austenitic structure is not realised even at finishing temperatures above the $\mathrm{Ar}_{3}$-temperature.

Although there is an obvious scientific and commercial significance, published research on the transformation from deformed austenite is limited in extent. ${ }^{5-11)}$ In addition, the majority of the published work describes either phenomenological observations or purely theoretical considerations without experimental verification. In many cases the principal aim of the research described is understanding the thermomechanical response of the material rather than understanding the transformation itself. Notwithstanding this, within the existing literature, there is a general consensus that deformation leads to accelerated transformation kinetics. However, what remains unclear is the exact nature of the mechanisms that drive this phenomenon.

The principal aims of the present paper are to separate more clearly the roles of nucleation and growth kinetics to understand better the mechanisms in operation, and to extend a mean field interface mobility control transformation model to describe the transformation kinetics from deformed austenite. Experimental results on the effect of plastic deformation on the austenite to ferrite transformation as manifest in the dilatometric signal during continuous cooling transformation experiments are presented. The continuous cooling transformation experiments were conducted after several degrees of plastic deformation had been imposed on the austenitic structure. An existing transformation model, which is essentially based on interface mobility concepts, ${ }^{3,4,12)}$ has been extended in order to account for the additional driving force arising from the stored energy of deformation. The nature of the deformation substructure and the effects of recovery are also taken into account.

\section{Background}

\subsection{The Effects of Deformation on the Transforma- tion Kinetics}

The effect of plastic deformation on the austentie $(\gamma)$ to ferrite $(\alpha)$ transformation kinetics is generally acknowl- 
edged as an accelerating effect. This has been observed in the different parameters that reflect the transformation kinetics. More specifically, in cooling experiments both the transformation start temperature and the transformation finish temperature have been found to increase, ${ }^{7,9)}$ the temperature range of the transformation to contract, ${ }^{9)}$ the final fraction of polygonal ferrite to increase ${ }^{5)}$ and the final ferrite grain size to decrease. ${ }^{7-9)}$ However, the exact nature of the effects that plastic deformation of austenite has on the transformation kinetics is poorly understood. A theoretical contribution by Umemoto et al. $^{6}{ }^{6}$ postulates that three different mechanisms may affect ferrite nucleation, whilst a fourth mechanism affects ferrite grain growth. The mechanisms postulated by Umemoto et al. are: (1) accelerated nucleation kinetics as a result of an increase in grain boundary area; (2) increased nucleation potency at grain boundaries via the introduction of grain boundary ledges leading to localised increases in grain boundary energy; (3) additional nucleation sites due to a deformation substructure; (4) accelerated growth kinetics arising from the stored energy of deformation. The first three mechanisms help overcome the energy barrier for nucleation leading to a reduction of the undercooling required to bring about nucleation in a cooling experiment, and possibly also to an increase in the nucleation site density. These mechanisms thus provide plausible explanations for the observations of increased transformation start and finish temperature, and also for the refinement of microstructure. The stored energy that gives rise to mechanism (4) essentially increases the driving force for the transformation and may explain the contraction of the transformation range. Expressed in microstructural terms, the stored energy of deformation is not only a function of the dislocation density but is also dependent upon the nature of the substructure in which the dislocations are stored. Experimental observations which suggest that deformation affects the nucleation behaviour are commonly reported. ${ }^{6}$ Experimental evidence of the influence of plastic deformation on the growth kinetics is less common. In fact it is often assumed that the effect on growth kinetics is negligible and Umemoto et al., ${ }^{10)}$ have shown experimentally that the thickening rate (the growth rate normal to the boundary into the grain centre) is unaffected by strain. However, whilst this result has been corroborated by Torizuka et al. ${ }^{11)}$ it remains nevertheless unclear whether this is broadly true for all conditions of strain, undercooling and nucleation pattern.

The magnitude of the effect of deformation is of course strongly dependent on the deformation conditions (strain rate, strain and temperature) via their effect on the nature and scale of the substructure and the dislocation density. However, due to the extreme experimental difficulties associated with observation of deformation substructure in austenite (the substructure is always destroyed on cooling to room temperature) little is known about its true nature. Quantitative estimates of the characteristics of the dislocation structure in austenite may be based on the many findings reported in literature for other FCC systems (principally $\mathrm{Ni}$ and $\mathrm{Al}$ ), and more recently findings reported for $\mathrm{Ni} / \mathrm{Fe}$ based model austenite systems. ${ }^{13-16)}$ These reports suggest that only at low strains do dislocations remain homogeneously distributed. Such homogeneous dislocation distrib- utions may not be expected beyond the first stages of work hardening (typically at true strains of up to 0.1 ). At high strains, in the steady state region of the stress-strain curve, the substructure can be expected to comprise of dislocation cells with low dislocation density interiors (typical cell sizes range from $0.5-1.0 \mu \mathrm{m}$, whilst the dislocation density in the cell body may be of the order of $10^{8} \mathrm{~m}^{-2}$ ). In a recent publication on the substructural development in a $\mathrm{Ni}-30 \% \mathrm{Fe}$ model austenite system Adachi et al. ${ }^{13)}$ estimate the total dislocation density after a reduction of $70 \%$ at $800^{\circ} \mathrm{C}$ to be approximately $1.5 \times 10^{14} \mathrm{~m}^{-2}$.

\subsection{The Transformation Model}

The model used here is a physically based transformation model validated for plain carbon steels which utilises both diffusivity and interface mobility concepts to describe the growth of ferrite. ${ }^{3,4,12)}$ The velocity of the austenite $(\gamma) /$ ferrite $(\alpha)$ interface is translated to global transformation kinetics (the increase in ferrite fraction with time) using a geometrical representation of the austenitic microstructure. The representative geometry used is that of the tetrakaidecahedron. ${ }^{17)}$

In the current model no detailed emphasis has been placed on the kinetics of nucleation. However the nucleation behaviour is described through two parameters: the nucleation undercooling $\Delta T$, and the nucleation site density $n_{\mathrm{n}}$. It is assumed that nucleation occurs instantaneously at a given temperature $T_{\mathrm{s}}$, usually chosen to optimise agreement with experimental data, and which may represent an undercooling below the equilibrium transformation start temperature, given by $\Delta T=T_{\mathrm{A} 3}-T_{\mathrm{s}}$. The nucleation site density is determined from the observed microstructures, taken as the number ratio of ferrite grains to austenite grains. The assumption that every nucleated ferrite grain survives in the transformed microstructure, and hence that no coarsening occurs, is implicit in this approach. Despite the simplicity of this treatment of nucleation, if $\Delta T$ and $n_{\mathrm{n}}$ are used as free parameters, mechanisms driving and enhanced nucleation site density and a reduction in undercooling for nucleation can be distinguished.

The basic assumption of the model is that the velocity of the $\gamma / \alpha$-interface is proportional to the local driving force for transformation, which is dependent on the local diffusion characteristics at a given point in the transformation. The proportionality factor is the interface mobility, which is governed by thermally activated processes. The velocity $v$ of the interface is then given by the expression

$$
v=M_{0} \exp \left(-\frac{Q}{R T}\right) \Delta G^{\alpha \gamma},
$$

in which $R$ is the gas constant, $T$ the temperature, and the three basic quantities which describe the kinetics of the transformation are the free energy difference $\Delta G^{\alpha \gamma}$, which acts as the driving force for the transformation, the activation energy $Q$ for atomic motion at the interface, and the pre-exponential factor $M_{0}$. In the case of the transformation model the driving force is taken to be the chemical free energy as obtained from the MTDATA thermodynamics database programme at different compositions at the interface and at different temperatures during the transformation. In 
the calculations it is assumed that the carbon content in either phase is homogeneous at any given time (mean-field approach). Since the temperature ranges involved are too narrow to determine both $M_{0}$ and $Q$ with sufficient accuracy, the value of $Q$ is taken from earlier work on Fe-Mn alloys ${ }^{12)}$ as $140 \mathrm{~kJ} \mathrm{~mol}^{-1}$, which is similar to the value found for recrystallisation in pure iron. ${ }^{18)}$ The model transformation curves (ferrite fraction as a function of time and temperature) are calculated by introducing the appropriate number of nuclei, with a density $n_{\mathrm{n}}$, at suitable sites within the tetrakaidecahedron (grain corners, edges, faces and centres may all be used) at the nucleation temperature $T_{\mathrm{s}}$. The nuclei subsequently grow spherically according to Eq. (1) and impingment effects at later stages of the process are properly taken into account. At every time step, the ferrite volume fraction in the grain is determined by numerical integration.

\subsection{Incorporation of Deformation Effects}

In order to extend the model to account for the effect of plastic deformation on austenite grain geometry, the dimensions of the tetrakaidecahedron along the three principal axes can be changed. A compression deformation, as employed in the present experiments, can be taken into account by "flattening" the tetrakaidecahedron along the $z$ axis (i.e. simple axial compression). ${ }^{19)}$ Furthermore, in the case of transformation from deformed austenite the total driving force $\Delta G^{\alpha \gamma}$ is expressed as the summation of the chemical driving force $\Delta G_{\text {chem }}$ and the additional driving force arising from the stored energy of deformation $\Delta G_{\text {def }}$, resulting in the equation

$$
\Delta G^{\alpha \gamma}=\Delta G_{\text {chem }}+\Delta G_{\text {def }} .
$$

In order to evaluate $\Delta G_{\text {def }}$ a number of assumptions must be made regarding the dislocation arrangement in the deformation substructure. Here, it is assumed that the deformation substructure is like that described by Adachi et al. ${ }^{13)}$ for $\mathrm{Ni}-30 \% \mathrm{Fe}$ deformed above $800^{\circ} \mathrm{C}$ comprising simply of cells/subgrains, and that dislocations are divided between two principal substructural components, dislocation-dense cell walls/subgrain boundaries and cell/subgrain interiors with a low dislocation density. Implicit in this approach is the assumption that heterogeneities in the substructure (for example microbands and shear bands) either do not occur or do not significantly contribute to the stored energy of deformation which is available for driving growth kinetics. This assumption can be defended since shear bands are known to accommodate high strains due to the ease with which they soften ${ }^{20)}$ (thus implying that the internal structure readily dynamically recovers or recrystallises and is therefore not densely dislocated), are likely to be narrow (thus occupy a limited volume) and, due to their large misorientation, provide attractive sites for the nucleation of ferrite $^{6,13)}$ (thus play a limited role in the growth process). If assumed to be cellular or subgranular, the substructure can be described using two principal parameters, the free dislocation density $\left(\rho_{\mathrm{i}}\right)$ and the cell/subgrain diameter $(\delta)$. The total stored energy of the dislocation substructure then becomes

$$
\Delta G_{\text {def }}=\frac{1}{2} \mu b^{2} \rho_{\mathrm{i}}+\frac{3 \sigma}{\delta},
$$

where $\mu$ is the shear modulus (taken to be $80 \mathrm{GPa}$ ), $b$ is the length of the Burgers vector $(0.25 \mathrm{~nm})$ and $\sigma$ is the subboundary interfacial energy (in all cases assumed to be 200 $\mathrm{mJ} \mathrm{m}^{-2}$ ).

A further complication when accounting for the effects of deformation is that in principle, the stored energy which may be responsible for accelerating the ferrite nucleation and growth kinetics, also provides the driving force for the softening processes of recovery and recrystallisation. In this sense recovery, recrystallisation and transformation can be considered to be competitive processes. In the present model recovery has been accounted for by two basic mechanisms, dislocation annihilation by thermally activated climb and subgrain coarsening. For the former process the annihilation rate is envisaged to be proportional to $\rho_{\mathrm{i}}$, which leads to an exponential decay of dislocation density according to

$$
\rho_{\mathrm{i}}=\rho_{\mathrm{i}, 0} \exp \left(-\frac{t}{\tau_{\rho}}\right),
$$

where $\tau_{\rho}$ is the relaxation time for the interior dislocation density, which is temperature dependent through a Boltzmann factor with an activation energy $Q_{\rho}$, with a preexponential factor $\tau_{0 \rho}$. According to this expression $\rho_{\mathrm{i}}$ tends to zero as $t \rightarrow \infty$. Cell coarsening is envisaged to obey the relationship

$$
\delta=\delta_{0}\left(1+\frac{t}{\tau_{\delta}}\right)^{1 / 2},
$$

according to Sæter and Nes, ${ }^{21)}$ where $\tau_{\rho}$ is the relaxation time for the cell growth, with an activation energy $Q_{\delta}$, and a pre-exponential factor $\tau_{0 \rho}$. Here, $\delta$ tends to infinity as $t \rightarrow \infty$. The effect of the recovery behaviour on $\Delta G_{\text {def }}$ is visualised in Fig. 1, which shows the gradual decrease of the two components of $\Delta G_{\text {def }}$ as a function of time, represented relative to the relaxation times. The evolution of both components with time is equivalent. Thus in practice the two recovery processes influence the transformation in the same way and cannot be seperated.

In principle, a deformed austenitic microstructure gives rise to a shift in the relative stability of the $\alpha$ - and $\gamma$-phases in the case that ferrite is undeformed. This effect is shown in Fig. 2, showing the total driving force $\Delta G^{\alpha \gamma}$ in a temperature interval near the equilibrium temperature for three conditions: for a non-deformed austenitic microstructure (i.e. $\rho_{\mathrm{i}}=0$ and $\delta=\infty$ ), for a deformed microstructure with a homogeneous dislocation density $\left(\rho_{\mathrm{i}}=1 \times 10^{15} \mathrm{~m}^{-2}\right.$ and $\delta=\infty)$ and for a deformed microstructure with a pure cell structure $\left(\rho_{\mathrm{i}}=0\right.$ and $\left.\delta=0.2 \mu \mathrm{m}\right)$. The temperature at which ferrite and austenite are in equilibrium, $T_{\mathrm{A} 3}$, is shifted to higher temperatures due to the additional free energy of the deformed austenite phase $\Delta G_{\text {def }}$. However, it should be noted that at temperatures above $T_{\mathrm{A} 3}$ for undeformed austenite the driving force for recrystallisation is larger than the driving force for transformation. Curve [2] in Fig. 2 illustrates the effect of recovery on $\Delta G^{\gamma \alpha}$ for $d T / d t=$ $-1 \mathrm{~K} \mathrm{~s}^{-1}$, through the mechanism of cell coarsening (Eq. 


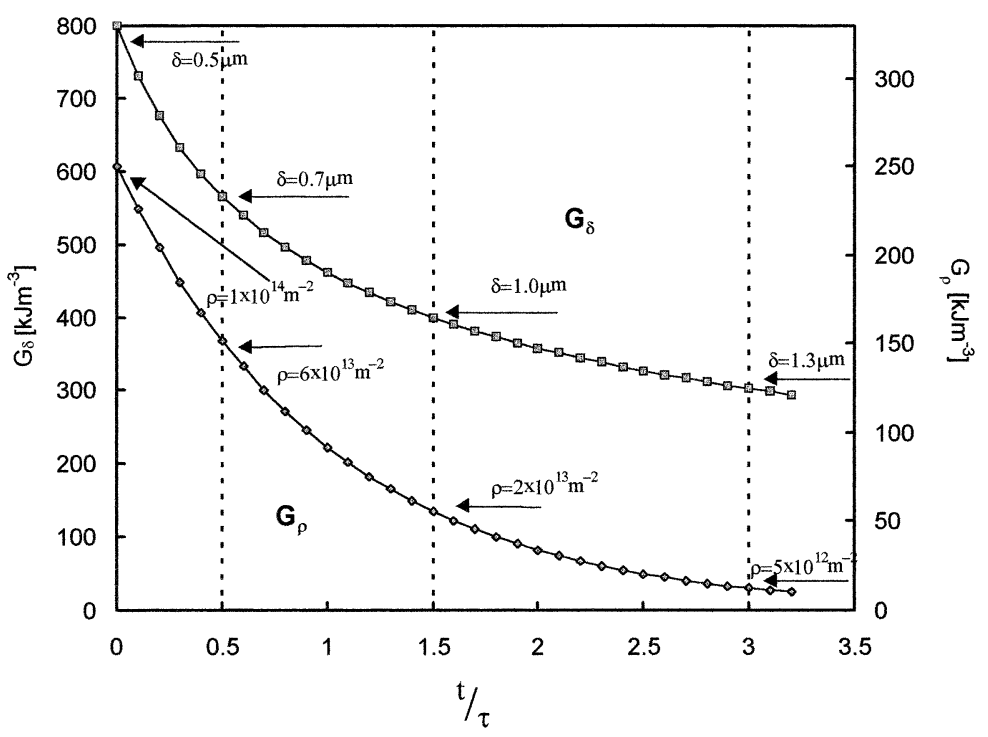

Fig. 1. The variation of the contributions to stored energy from free dislocations $\left(G_{\rho}\right)$ and the cell walls $\left(G_{\delta}\right)$ during the recovery processes described in Eqs. (4) and (5). The initial values have been arbitrarily chosen at $\rho_{\mathrm{i} 0}=10^{14} \mathrm{~m}^{-2}$ and $\delta_{0}=0.5 \mu \mathrm{m}$ ( $\tau$ represents the relaxation times for both the dislocations and the cell structure).

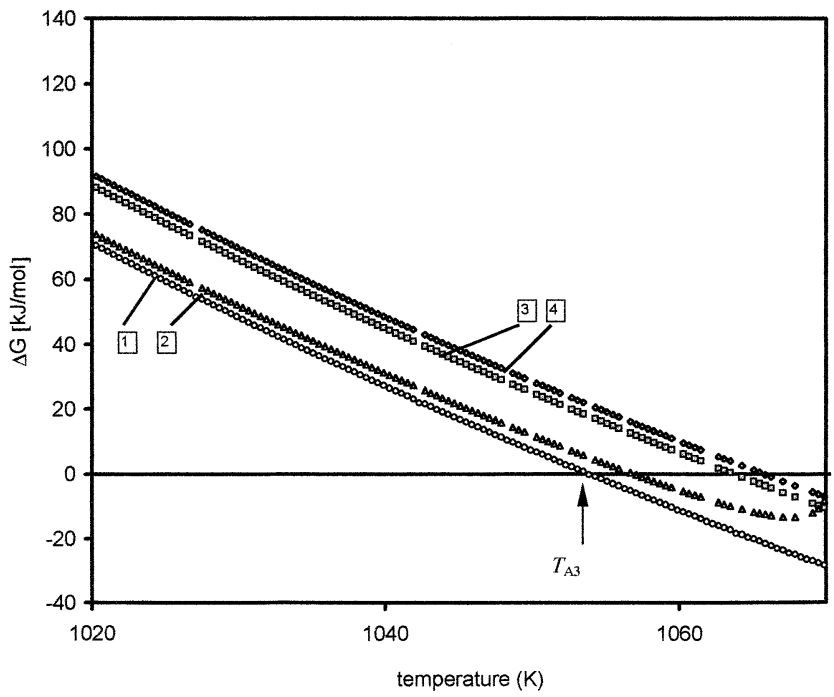

Fig. 2. Graph comparing the variation of the driving force for transformation expected for an undeformed system [1], with that for deformed systems containing a homogeneous distribution of dislocations [3] and a well developed cell/subgrain structure [4]. The effect of recovery of the subgrain structure on the driving force for transformation is also illustrated [2]. The initial values of dislocation density and subgrain diameter have been arbitrarily chosen at $\rho_{\mathrm{i} 0}=10^{15} \mathrm{~m}^{-2}$ and $\delta_{0}=0.2 \mu \mathrm{m}$.

(5)) with $\tau_{0 \delta}=2 \cdot 10^{7} \mathrm{~s}$ and $Q_{\delta}=170 \mathrm{~kJ} / \mathrm{mol}$, which implies $\tau_{\delta} \approx 2 \mathrm{~s}$ over the temperature range considered.

In the present study the effects of pre-transformation deformation are interpreted in terms of the recovery behaviour. In reality both recovery and recrystallisation are likely to occur, but in the present interpretation recrystallisation will be disregarded. Effectively this means that the observations regarding recovery should be seen as the effective result of both recovery and recrystallisation which in any case both influence the transformation primarily through their effect on the same parameter, the stored energy of deformation.

\subsection{Modelled Effects of Deformation on the Transfor- mation Kinetics}

In order to show the effect of the stored energy of deformation on transformation kinetics a number of calculated transformation curves are shown (Figs. 3 and 4) in which either the homogeneous dislocation density $\rho_{\mathrm{i}}$ or the cell size $\delta$ is varied. For all transformation curves the cooling rate is $1 \mathrm{~K} \mathrm{~s}^{-1}$, the pre-exponential factor $M_{0}$ is 0.2 $\mathrm{molm} / \mathrm{Js}$, the number of nuclei is 6 per prior austenite grain, and the diameter of the tetrakaidecahedron representing the austenite grain is $50 \mu \mathrm{m}$ (i.e. a nucleus density of $\left.1.1 \times 10^{14} \mathrm{~m}^{-3}\right)$. Nucleation is assumed to take place instantaneously at $T=T_{\mathrm{A} 3}$ (i.e. the nucleation undercooling is zero). Unless indicated otherwise, the substructure has been assumed stable so that recovery does not take place.

Figure 3 illustrates the predicted effect of a homogeneous distribution of dislocations for several fixed values of $\rho_{\mathrm{i}}$ (no recovery), and for one case in which recovery takes place at a moderate rate $\left(Q_{\rho}=170 \mathrm{~kJ} \mathrm{~mol}^{-1}\right)$. The variation of the equilibrium ferrite fraction with temperature is also shown in Fig. 3. In agreement with Fig. 2, Fig. 3 shows that a theoretical consequence of the presence of stored energy of deformation is that the transformation start temperature may be elevated above the equilibrium start temperature. However, in practice, it is unlikely that the start temperature will exceed the equilibrium value for systems which undergo rapid softening. In such systems the mechanisms of recovery and recrystallisation are also driven by the stored energy of deformation Therefore, since they will lead to a reduction of the available stored energy, softening processes can be considered to be competitive with the transformation. Furthermore, the consumption of stored energy of deformation will naturally be greater at low cooling rates, where softening processes have more time to occur, whilst at higher cooling rates, where the time for recovery and recrystallisation is limited, the transformation may be expected to commence only after appreciable undercooling.

According to Fig. 3, only dislocation densities of $10^{14}$ $\mathrm{m}^{-2}$ and greater have a significant effect on the transforma- 


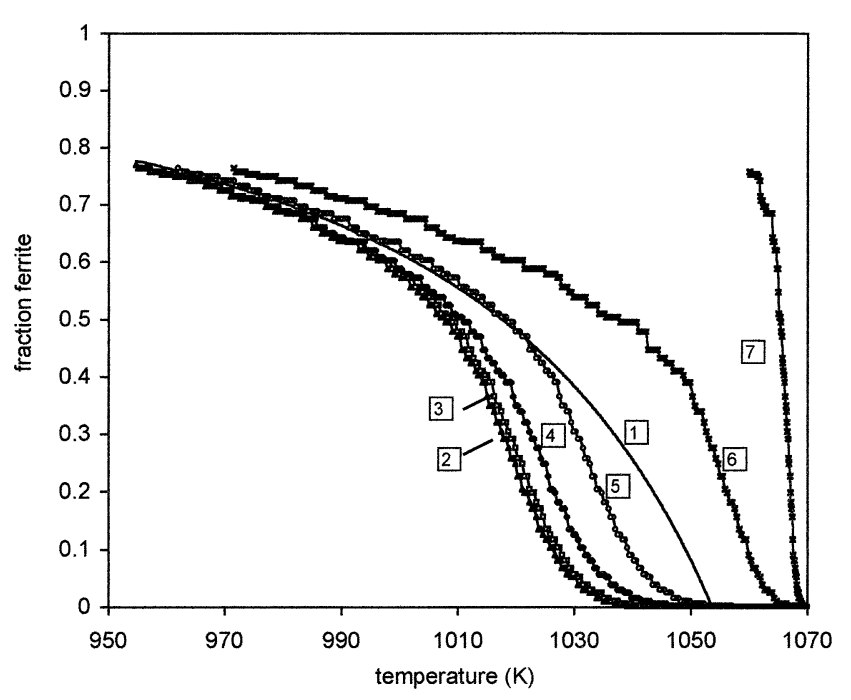

Fig. 3. Model transformation curves showing the effect of increasing dislocation density and concurrent recovery by dislocation anihilation on the kinetics of transformation: [1] equilibrium ferrite fraction, [2] no stored energy of deformation, [3] dislocation density $10^{14} \mathrm{~m}^{-2}$, [4] dislocation density $10^{15} \mathrm{~m}^{-2}$ with concurrent recovery, [5] dislocation density $10^{15} \mathrm{~m}^{-2}$, [6] dislocation density $3 \times 10^{15}$ $\mathrm{m}^{-2}$, [7] dislocation density $10^{16} \mathrm{~m}^{-2}$.

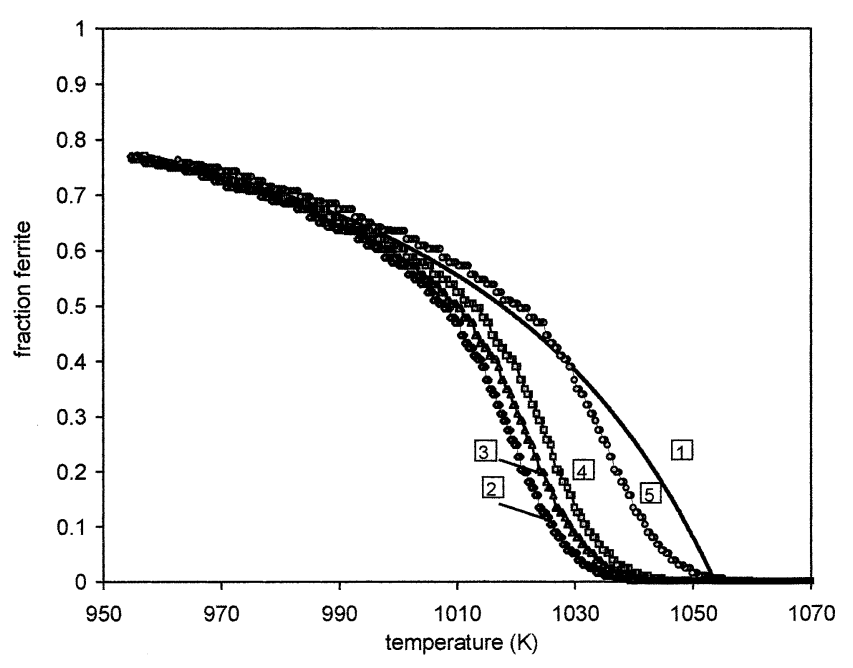

Fig. 4. Model transformation curves showing the effect of decreasing subgrain diameter and concurrent recovery by subgrain coarsening on the kinetics of transformation: [1] equilibrium fraction ferrite, [2] no free energy of deformation, [3] subgrain diameter of $0.2 \mu \mathrm{m}$ with concurrent recovery, [4] subgrain diameter $0.5 \mu \mathrm{m}$, [5] subgrain diameter $0.2 \mu \mathrm{m}$.

tion kinetics (for lower dislocation densities the curve is approximately identical to that calculated for a purely chemical driving force). For a dislocation density of $10^{15} \mathrm{~m}^{-2}$ there is a pronounced acceleration of the transformation, the most pronounced effect being the elevation of the transformation start temperature. In contrast, the transformation finish temperature is only marginally affected since the transformation curves converge at higher ferrite fractions. For a dislocation density of $10^{16} \mathrm{~m}^{-2}$ (i.e. two orders of magnitude higher than that expected from the experiments of Adachi et al. ${ }^{13)}$ ) an extremely rapid transformation takes place. In this case the transformation start temperature is driven above the equilibrium value and a pronounced in- crease in the transformation finish temperature is found.

In the case considered in Fig. 4 the dislocation substructure is assumed to be subgranular with a negligible internal dislocation density (in Fig. 3 dislocation densities of $10^{13}$ $\mathrm{m}^{-2}$ or lower have been shown to have no apparent effect on the predicted transformation curves). The model predicts that cell structures with diameters in the range $0.2-$ $1.0 \mu \mathrm{m}$ have an appreciable effect on the transformation start temperature (within this range of cell sizes the equilibrium temperature is never exceeded), whilst the transformation finish temperature is influenced to a lesser extent. At extremely small cell diameters $(\delta=0.2 \mu \mathrm{m}$, again lower than that expected from the literature ${ }^{13)}$ ) a moderate elevation of the transformation finish temperature is predicted. As in Fig. 3, the effect of recovery is shown in one of the curves in Fig. 4.

\section{Experimental}

The material chosen for the investigation is a $\mathrm{C}-\mathrm{Mn}$ steel with a chemical composition of $0.19 \mathrm{wt} \% \mathrm{C}, 1.46 \mathrm{wt} \% \mathrm{Mn}$, $0.445 \mathrm{wt} \% \mathrm{Si}$ and $0.010 \mathrm{wt} \% \mathrm{~S}$. This steel was chosen because the simple alloy chemistry is devoid of precipitates and strong solution strengthening additions. The high Mncontent was chosen in order to retard growth kinetics, thus making the experimental determination more accurate.

All deformation and dilatometry testing was conducted on a Bähr 805 dilatometer with a deformation unit designed to enable the imposition of a simple uniaxial deformation. The geometry of the samples used was, in all cases, cylindrical with a length of $10 \mathrm{~mm}$ and a diameter of $5 \mathrm{~mm}$. In all tests the temperature was measured using a surface mounted k-type thermocouple located at the mid length of the specimen. In a number of experiments the temperature was measured at two locations on the outer surface of the cylindrical sample, viz., in the middle and near to the end of the sample. The temperature differences thus registered were limited to approximately $5 \mathrm{~K}$.

In all cases an austenitisation heat treatment at $1273 \mathrm{~K}$ for $10 \mathrm{~min}$ is applied prior to cooling rapidly to the deformation temperature. In order to introduce a varying state of deformation into the austenitic microstructure prior to transformation, the deformation was performed at a range of strain rates $\left(0.1 \mathrm{~s}^{-1}, 1 \mathrm{~s}^{-1}, 10 \mathrm{~s}^{-1}\right)$ and at two different temperatures $(1123 \mathrm{~K}$ and $1173 \mathrm{~K})$. Each sample was deformed in compression up to a strain of 0.6. Immediately after the deformation the samples were cooled at a rate of $15 \mathrm{~K} \mathrm{~s}^{-1}$, while recording the dilatation signal. The dilatation data were interpreted in terms of the ferrite fraction as a function of temperature by means of a lever-rule method. $^{22)}$

Using optical microscopy and a thermal etching technique, ${ }^{23}$ the re-heated austenite grain size prior to deformation was determined by the linear-intercept method to be $30 \mu \mathrm{m}$. This value translates to a $3 \mathrm{D}$, tetrakaidecahedral diameter of approximately $50 \mu \mathrm{m} .{ }^{24)}$ To date it has not proven feasible to experimentally determine the post deformation austenite grain dimensions. After cooling to room temperature, all samples were sectioned longitudinally, ground, polished and etched in $2 \%$ nital. The transformed microstructures were characterised using a Neophot 30 optical micro- 


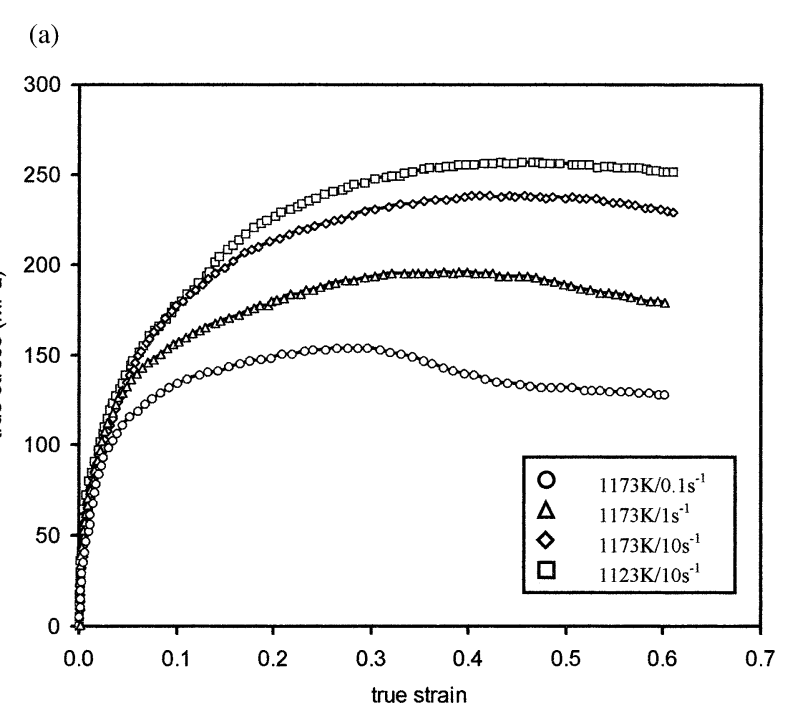

(b)

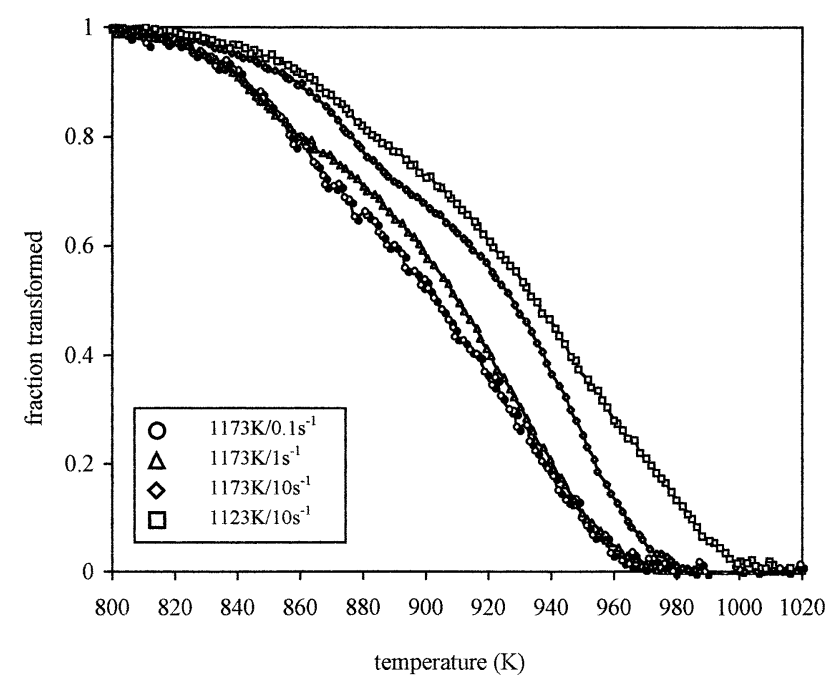

Fig. 5. The results of deformation dilatometry experiments: (a) true stress true strain curves for uniaxial compression deformations and (b) the fraction transformed as a function of temperature determined after the deformations shown in (a).

scope. In order to eliminate uncertainties arising from heterogeneities in deformation, care was taken to observe the structure in the same location in each sample. ${ }^{25}$ )

\section{Results and Discussion}

The deformation behaviour for the different temperatures and strain rates is shown in Fig. 5(a). In the stress-strain curves softening effects, which become more pronounced with decreasing strain rate and increasing temperature, can be clearly observed. The softening is indicative of dynamic recrystallisation the rate of which is dependent upon strain rate and temperature through the Zener-Holloman parameter. ${ }^{26)}$ From Fig. 5(a) it can be concluded that the deformation state, or the amount of stored energy at the end of the deformation treatment, increases in the order $\left[0.1 \mathrm{~s}^{-1}\right.$ at $1173 \mathrm{~K}],\left[1 \mathrm{~s}^{-1}\right.$ at $\left.1173 \mathrm{~K}\right],\left[10 \mathrm{~s}^{-1}\right.$ at $\left.1173 \mathrm{~K}\right],\left[10 \mathrm{~s}^{-1}\right.$ at $1123 \mathrm{~K}]$ as the extent of dynamic recrystallisation decreases. The effect of this increasing stored energy on the transformation kinetics can be seen in Fig. 5(b), which shows the transformation curves recorded during cooling immediately after the deformations depicted in Fig. 5(a). From these curves it is apparent that Both start and finish temperatures shift to higher temperatures with increasing stored energy of deformation.

The ferrite in the transformed microstructure was found to be much finer than the original austenitic structure. Mean linear intercept grain sizes of $15 \mu \mathrm{m}\left[0.1 \mathrm{~s}^{-1}\right.$ at $\left.1173 \mathrm{~K}\right]$, $13 \mu \mathrm{m}\left[1 \mathrm{~s}^{-1}\right.$ at $\left.1173 \mathrm{~K}\right], 12 \mu \mathrm{m}\left[10 \mathrm{~s}^{-1}\right.$ at $\left.1173 \mathrm{~K}\right]$, and $11 \mu \mathrm{m}\left[10 \mathrm{~s}^{-1}\right.$ at $\left.1123 \mathrm{~K}\right]$ were measured. These values yield nucleus density of the order of $10^{14}-10^{15} \mathrm{~m}^{-3}$.

In order to analyse the effect of deformation on the transformation kinetics, the transformation curves of Fig. 5(b) have been modelled by means of the interface-mobility model presented in Sec. 2. However, a number of parameters which are extremely difficult to determine experimentally play an important role in this model. Moreover, combined effects due to variations in different parameters may be hard to interpret. Therefore, an extensive study has been made of the influence of different parameters under the assumption of fixed values for others. A number of choices have been made in order to systematically model the transformation curves. Firstly, since the experimental determination of this quantity after deformation has not proven feasible, the austenite grain size is assumed to be that of the reheated structure (a value of $50 \mu \mathrm{m}$ ) for all deformation conditions. Secondly, the stored energy is expressed in terms of a subgrain diameter (i.e. the internal dislocation density was considered in all cases to be negligible). However, as can be seen in Figs. 1, 3 and 4, the effects of a homogeneous dislocation density and a subgrain structure on the stored energy are very similar. Furthermore, the decay of stored energy resulting from the two recovery mechanisms considered follows a similar path. Therefore, these phenomena can not readily be separated and consequently the choice to represent the stored energy and recovery through the variation of $\delta$ alone is justifiable. Finally, mobility of the transforming interface is assumed to be given by the parameter values $M_{0}=0.2 \mathrm{molm} / \mathrm{J} \mathrm{s}$ and $Q=140 \mathrm{~kJ} \mathrm{~mol}^{-1}$. These values are logical choices in view of previous studies, ${ }^{27)}$ and the constancy of these parameters is a consequence of the modelling approach, i.e. it is assumed that the interface velocity may be affected by the deformation state through $\Delta G_{\text {def }}$, but the mobility is not. These three assumptions leave three parameters to be considered in the modelling: the cell size $\delta$, the nucleus density $n_{\mathrm{n}}$, and the undercooling $\Delta T$.

In the first set of calculations, the nucleus density and the undercooling are given fixed values $\left(n_{\mathrm{n}}=8.6 \times 10^{14} \mathrm{~m}^{-3}\right.$ and $\Delta T=60 \mathrm{~K})$. The model transformation curves for optimal values of $\delta$ are shown in a comparison with the experimental curves in Fig. 6. It should be noted that the modelling only considers the ferrite formation (the final fraction is thus 0.8 ). The remainder of the process is pearlite formation. The effect of deformation on the transformation kinetics observed experimentally is not well reproduced under the assumption that the stored energy results in an increased interface velocity with unaffected nucleation behaviour. Increasing the stored energy of deformation results in an increased growth kinetic (an increase in the slope of the transformation curve) which is not apparent in the ex- 


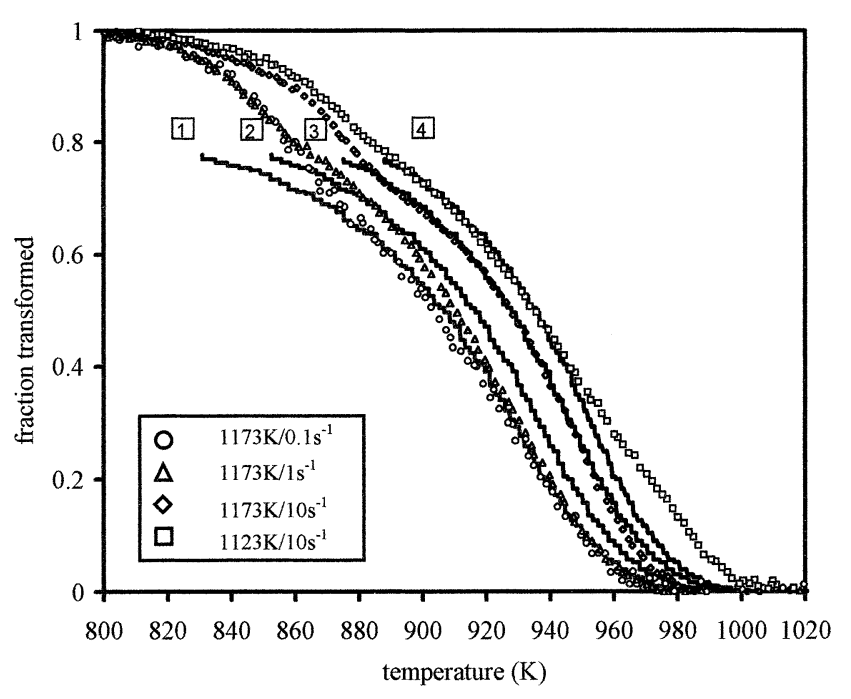

Fig. 6. A comparison of experimental (open symbols) and modelled (solid lines) transformation curves. The model curves shown represent the optimal fits to the experimental data which may be obtained by variation of the cell diameter whilst holding all other parameters constant. [1] No stored energy of deformation, [2] subgrain diameter $1 \mu \mathrm{m}$, [3] subgrain diameter $0.5 \mu \mathrm{m}$, [4] subgrain diameter $0.2 \mu \mathrm{m}$.

perimental data, particularly during the early stages of transformation. Furthermore, the magnitude of the displacement of the transformation curves for severe deformations can only be reproduced by inputting cell sizes which are unrealistically small (i.e. $0.2 \mu \mathrm{m}$ whereas cell sizes are reported to be typically $1-0.5 \mu \mathrm{m}$ ). It should also be noted that the value of boundary energy $(\sigma)$ adopted for these calculations is $0.2 \mathrm{~J} \mathrm{~m}^{-2}$. This is a value which may be expected for a low angle boundary of approximately $4^{\circ}$. This represents the expected upper limit of cell boundary misorientation. This will inevitably lead to a result which represents an upper bound for the effects under scrutiny. The discrepancies between the model and the experimental curves are such that "fine-tuning" the model curves by introducing recovery effects is not expected to lead to a satisfactory agreement either (cf. Fig. 4). From extensive series of calculations with different parameter values it is concluded that the behaviour seen in Fig. 6 is not caused by the specific values chosen for either $\delta$ or the other parameters, but is general. From these results it would appear possible to draw the general conclusion that the experimental data cannot be adequately described by an effect on growth alone.

As a second parameter to be investigated, the nucleus density $n_{\mathrm{n}}$ has been considered. In principle the nucleus density can be deduced from the microscopical observations on the ferritic microstructure. When the austenitic and ferritic grain diameters are given by $d^{\gamma}$ and $d^{\alpha}$, respectively, in the model austenite grain a number of $\left(d^{\gamma} / d^{\alpha}\right)^{3}$ should be used for the number of nuclei per austenite grain. However, due to the lack of information regarding $d^{\gamma}$ after deformation, and the discrepancies between $\left(d^{\alpha}\right)^{-3}$ and $n_{\mathrm{n}}$ due to possible ferrite grain coarsening, ${ }^{26)}$ it is chosen to treat $n_{\mathrm{n}}$ as a freely variable parameter. An estimate of the likely range of nucleation site densities was, however, obtained from the ratios of the re-heated austenite grain volume to the final ferrite grain volume. The effect of varying $n_{\mathrm{n}}$ within this

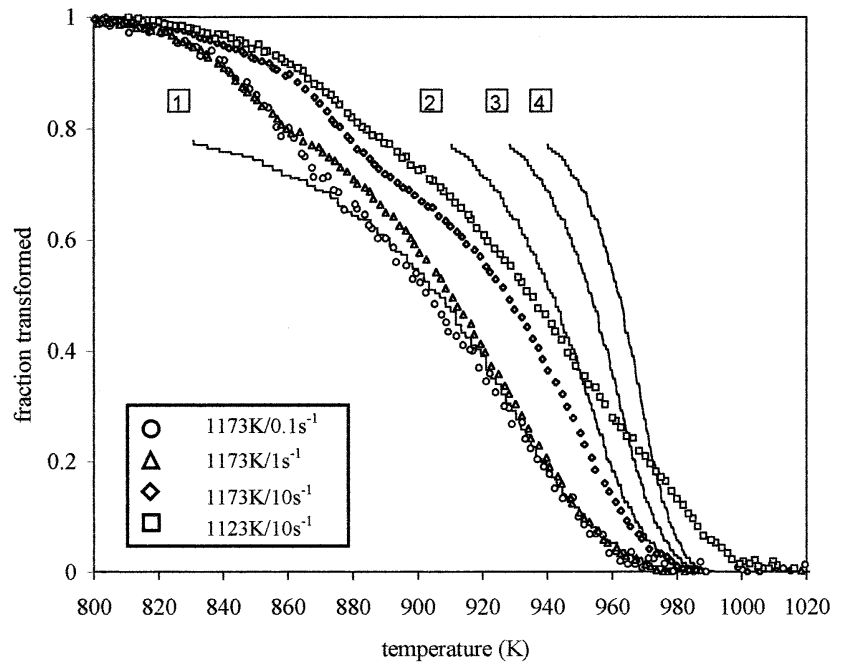

Fig. 7. A comparison of experimental (open symbols) and modelled (solid lines) transformation curves. The model curves shown represent the predicted variation obtained when the nucleation site density is varied within experimentally estimated limits whilst all other parameters are held constant. [1] Nucleation site density $8.6 \times 10^{14} \mathrm{~m}^{-3}$, [2] nucleation site density $1.4 \times 10^{15} \mathrm{~m}^{-3}$, [3] nucleation site density $1.7 \times 10^{15} \mathrm{~m}^{-3}$, [4] nucleation site density $2.0 \times 10^{15} \mathrm{~m}^{-3}$.

range is shown in the model curves of Fig. 7, with the accompanying parameter values $\delta=\infty \mu \mathrm{m}$ (free energy of deformation was held at 0 ) and $\Delta T=60 \mathrm{~K}$. This choice of parameters means that the effect of deformation is envisaged to occur as an increase in the density of effective nucleation sites, with neither the nucleation kinetics themselves nor the interface velocity being affected. Also in this case no satisfactory reproduction of the experimental transformation curves was obtained. This observation is entirely compatible with the general observation in the literature that deformation banding is predominatly responsible for providing additional intragranular nucleation sites. Based on the observations of Adachi et al. ${ }^{13)}$ such band structures may not be expected for the deformation conditions imposed here.

Finally, the undercooling $\Delta T$ was taken as the free parameter. For $\delta$ and $n_{\mathrm{n}}$ the values $\delta=\infty$ and $n_{\mathrm{n}}=8.6 \times 10^{14} \mathrm{~m}^{-3}$ were chosen. The results are presented in Fig. 8. It is clear that this set of model calculations gives the best reproduction of the experimental transformation curves since the transformation curves are displaced and do not exhibit a change in slope. Therefore, whilst an acceleration of the growth kinetics cannot be entirely ruled out, the effect of deformation on the transformation kinetics is thought to be predominantly due to an enhanced nucleation behaviour, i.e. a reduction of the undercooling necessary to induce nucleation. From a thermodynamical point of view, this conclusion means that the additional driving force $\Delta G_{\text {def }}$ leads to a reduction of the activation energy for nucleation. Consequently, an activation energy low enough to cause appreciable nucleation is reached at lower values for the undercooling. This is consistent with a mechanism which results in an increased potency of grain boundaries as nucleation sites. Such mechanisms have been proposed by several authors. Umemoto et al. postulate that enhanced nucle- 


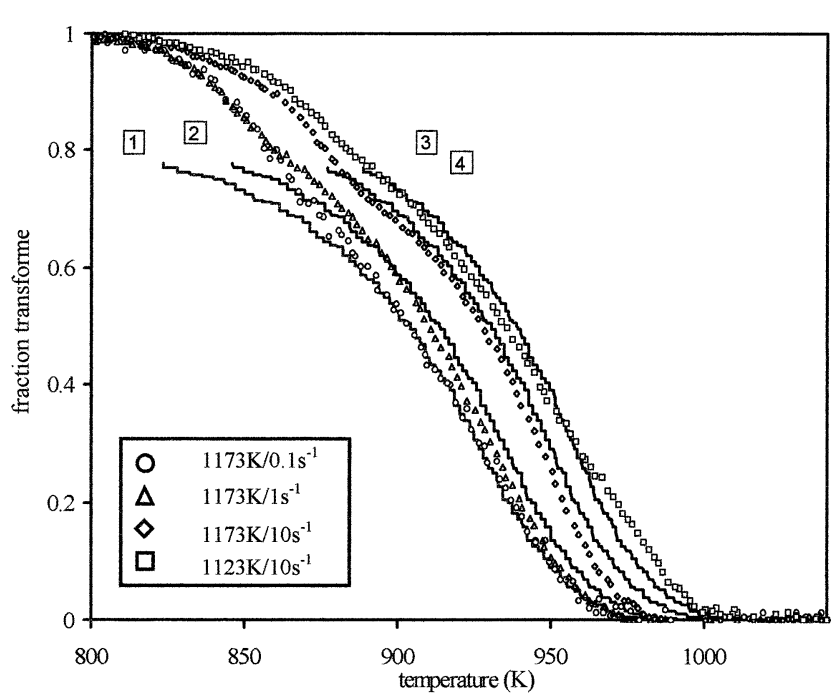

Fig. 8. A comparison of experimental (open symbols) and modelled (solid lines) transformation curves. The model curves shown represent the optimal fits to the experimental data which may be obtained by variation of the undercooling required for nucleation whilst holding all other parameters constant. [1] Undercooling $60 \mathrm{~K}$, [2] undercooling $55 \mathrm{~K}$, [3] undercooling $40 \mathrm{~K}$, [4] undercooling $30 \mathrm{~K}$.

ation is facilitated by grain boundary steps which form at the intersection of shear bands and grain boundaries. ${ }^{6}$ ) Similarly Roberts et ll. $^{28)}$ propose a mechanism based on grain boundary curvature which results from the accomodation of local stresses arising from a cell structure. ${ }^{28)}$ The observations of Adachi et al. ${ }^{13)}$ (and the observations reported here) that deformation banding may not be expected under the experimental conditions imposed here would imply that the latter mechanism proposed by Roberts ${ }^{28)}$ is the most probable.

The number of parameters involved in the modelling is too large to reach a quantitative determination of all parameters with an acceptable level of accuracy. Nevertheless, a useful analysis can be made on the basis of the effect of each of the parameters with the use of fixed values for others. As stated before, the effects of $\delta$ and $\rho_{\mathrm{i}}$ are equivalent and can be gathered together in one of the two parameters. Likewise, the effect of the austenite grain size prior to transformation is (mathematically) strongly linked to variations in the nucleus density and the mobility. The undercooling $\Delta T$ is apparently the parameter that is least correlated with others, and therefore the conclusion from the present study that the effect of deformation on the transformation kinetics is primarily caused by a decrease of the activation energy for nucleation, seen as a reduced undercooling, seems justified.

\section{Conclusions}

A series of dilatometric experiments on a plain carbon steel grade having undergone plastic deformation in the austenitic phase reveals a distinct effect of the degree of deformation on the transformation kinetics. Less softening during the deformation leads to a shift to higher temperatures, of the temperature range over which the transformation of austenite to ferrite takes place.
A modeling approach to account for the effect of deformation on the austenite to ferrite transformation has been constructed based on interface mobility concepts. In this approach the stored energy of deformation resulting from the formation of a dislocation substructure (represented here by two parameters; the dislocation density and the subgrain size) is considered to result in an elevation of the driving force for transformation. A scheme which accounts for a reduction in the stored energy available for driving the transformation as the result of the recovery is also incorporated. The model treats nucleation simply, assuming instantaneous site saturation, but incorporates two adjustable parameters which enable the effects of changes in nucleation site density and nucleation undercooling to be separated.

The model indicates that, particularly at low undercoolings and for high applied strains, deformation may have a pronounced influence on ferrite growth kinetics. However, it has also been shown that the occurrence of recovery and recrystallisation may severely limit this effect.

Comparison of modelled and experimental transformation curves indicates that deformation affects the transformation principally via a reduction in the undercooling required for nucleation rather than via an acceleration of the growth kinetics or a change in the nucleation site density.

\section{Acknowledgements}

The continued interest of CORUS R, D \& T, I Jmuiden is greatfully acknowledged.

\section{REFERENCES}

1) C. Zener: J. Appl. Phys., 20 (1949), 950.

2) M. Enomoto: Proc. Int. Conf. on Physical Metallurgy of Thermomechanical Processing of Steels and Other Metals (THERMEC 88), ISIJ, Tokyo, (1988), 360.

3) J. W. Christian: The Theory of Transformations in Metals and Alloys, 2nd ed., Pergamon Press, Oxford, (1981).

4) G. P. Krielaart, J. Sietsma and S. van der Zwaag: Mater. Sci. Eng., 237A (1997), 216.

5) A. J. De Ardo: Conf. Proc. Accelerated Cooling of Steels, ed. by P. Southwick, AIME, Pittsburgh, PA, (1986), 97.

6) M. Umemoto, A. Hiramatsu, A. Moriya, T. Watanabe, S. Nanba, N. Nakajima, G. Anan and Y. Higo: ISIJ Int., 32 (1992), 306.

7) X. Liu, L. P. Karjalainen and J. S. Pertulla: Conf. Proc. 2nd Int. Conf. on Modelling of Metal Rolling Processes, ed. by J. H. Beynon, P. Ingham, H. Teichert and K. Waterson, The Institute of Metals, London, (1996), 489.

8) M. J. Crooks, A. J. Garrat-Reed, J. B. Vander Sande and W. S. Owen: Metall. Trans., 13A (1982), 1347.

9) R. Pandi, M. Militzer, E. B. Hawbolt and T. R. Meadowcroft: Conf. Proc. 37th MWSP, Vol. XXXIII, ISS, Warrendale, PA, (1996), 635.

10) M. Umemoto, H. Ohtsuka, H. Kato and I. Tamura: Conf. Proc. on High Strength Low Alloy Steels, ed. by D. P. Dunne and T. Chandra, Univ. of Wollongong, Wollongong, (1984), 107.

11) S. Torizuka, O. Umezawa, K. Tsuzaki and K. Nagai: Tetsu-toHagané, 86 (2000), 26.

12) G. Krielaart and S. van der Zwaag: Mater. Sci. Technol., 14 (1998), 10 .

13) Y. Adatchi, T. Tomida and S. Hinotani: ISIJ Int., 40 (2000), Suppl., S194.

14) P. J. Hurley, B. C. Muddle, P. D. Hodgson, C. H. J. Davies, B. P. Wyne, P. Cizek and M. R. Hickson: Mater. Sci. Forum, 284-286 (1998), 159.

15) B. Bay, N. Hansen, D. A. Hughes and D. Kuhlmann-Wilsdorf: Acta Metall. Mater., 40 (1992), 205.

16) D. A. Hughes and N. Hansen: Mater. Sci. Technol., 7 (1991), 544.

17) Y. van Leeuwen, S. Vooijs, J. Sietsma and S. van der Zwaag: Metall. Mater. Trans., 29A (1998), 2925. 
ISIJ International, Vol. 41 (2001), No. 9

18) M. Hillert: Metall. Trans., 6A (1975), 5 .

19) S. B. Singh and H. K. D. H. Bhadesia: Mater. Sci. Technol., 14 (1998), 832.

20) S. Wierzbinski, A. Korbel and J. J Jonas: Mater Sci. Technol., 8 (1992), 153.

21) J. A. Saete, B. Forbord, H. E. Vatne and E. Nes: Conf. Proc., ICAA 6, The Japanese Institute for Light Metals, Tokyo, (1998), 113.

22) M. Onink, F. D. Tichelaar, C. M. Brakman, E. J. Mittemeijer and S. van der Zwaag: Z. Metallkd., 87 (1996), 24.

23) R. Riedle: Metallography, 14 (1981), 119.

24) A. R. Guimelli, M. Militzer and E. B. Hawbolt: ISIJ Int., 39 (1999),
271.

25) D. N. Hanlon, J. Sietsma and S. van der Zwaag: Conf. Proc., MMRP 3, The Institute of Materials, London, (1999), 431.

26) L. P. Karjalainen, J. A. Koskiniemi and X. D. Liu: 37th Mechanical Working and Steel Processing Conf. Proc., Vol. XXXIII, ISS Warrendale, PA, (1996), 861.

27) T. A. Kop, Y. van Leeuwen, J. Sietsma and S. van der Zwaag: ISIJ Int., 40 (2000), 713.

28) W. Roberts, H. Lidefelt and A. Sandberg: Conf. Proc., Hot Working and Forming Processes, Eds. by C. M. Sellars and G. J. Davies, Metals Society, London, (1980), 38. 